\title{
BMJ Open Innovative public-private partnership to target subsidised antimalarials: a study protocol for a cluster randomised controlled trial to evaluate a community intervention in Western Kenya
}

\author{
Jeremiah Laktabai, ${ }^{1}$ Adriane Lesser, ${ }^{2}$ Alyssa Platt, ${ }^{2,3}$ Elisa Maffioli, ${ }^{2,4}$ \\ Manoj Mohanan, ${ }^{2,4,5}$ Diana Menya, ${ }^{6}$ Wendy Prudhomme O'Meara, ${ }^{2,6,7}$ \\ Elizabeth L Turner ${ }^{2,3}$
}

To cite: Laktabai J, Lesser A, Platt $A$, et al. Innovative public-private partnership to target subsidised antimalarials: a study protocol for a cluster randomised controlled trial to evaluate a community intervention in Western Kenya. BMJ Open 2017;7: e013972. doi:10.1136/ bmjopen-2016-013972

- Prepublication history and additional material is available. To view please visit the journal (http://dx.doi.org/ 10.1136/bmjopen-2016013972).

Received 23 August 2016 Revised 23 December 2016 Accepted 12 January 2017

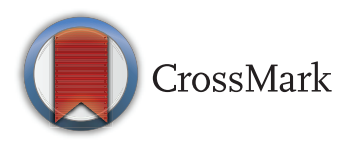

For numbered affiliations see end of article.

Correspondence to Dr Elizabeth L Turner; liz.turner@duke.edu

\section{ABSTRACT}

Introduction: There are concerns of inappropriate use of subsidised antimalarials due to the large number of fevers treated in the informal sector with minimal access to diagnostic testing. Targeting antimalarial subsidies to confirmed malaria cases can lead to appropriate, effective therapy. There is evidence that community health volunteers (CHVs) can be trained to safely and correctly use rapid diagnostic tests (RDTs). This study seeks to evaluate the public health impact of targeted antimalarial subsidies delivered through a partnership between $\mathrm{CHVs}$ and the private retail sector. Methods and analysis: We are conducting a stratified cluster-randomised controlled trial in Western Kenya where 32 community units were randomly assigned to the intervention or control (usual care) arm. In the intervention arm, CHVs offer free RDT testing to febrile individuals and, conditional on a positive test result, a voucher to purchase a WHOqualified artemisinin combination therapy (ACT) at a reduced fixed price in the retail sector.

Study outcomes in individuals with a febrile illness in the previous 4 weeks will be ascertained through population-based cross-sectional household surveys at four time points: baseline, 6, 12 and 18 months postbaseline. The primary outcome is the proportion of fevers that receives a malaria test from any source (CHV or health facility). The main secondary outcome is the proportion of ACTs used by people with a malaria-positive test. Other secondary outcomes include: the proportion of ACTs used by people without a test and adherence to test results.

Ethics and dissemination: The protocol has been approved by the National Institutes of Health, the Moi University School of Medicine Institutional Research and Ethics Committee and the Duke University Medical Center Institutional Review Board. Findings will be reported on clinicalstrials.gov, in peer-reviewed publications and through stakeholder meetings including those with the Kenyan Ministry of Health.

Trial registration number: Pre-results, NCT02461628.

\section{Strengths and limitations of this study}

- This protocol describes a robust study design: stratified randomised allocation contributes to good internal validity and outcome measures are collected independently of the intervention implementation.

- The study is conducted in a rural region of Western Kenya and is representative of other malaria-endemic regions in East Africa, which promotes external validity.

- The public-private partnership intervention evaluated in this study was developed using outcomes from our related pilot study and has cross-sectoral support.

- The intervention draws on both an existing community health volunteer system and a robust medicine retail sector. In the absence of either element, implementation could be challenging in other settings.

\section{INTRODUCTION}

In most malaria-endemic countries, including Kenya, a large fraction of fevers are treated in the informal health sector at retail medicine outlets such as chemists, pharmacists and small, unregulated medicine shops. ${ }^{1-5}$ These retail outlets are often found in more accessible locations than formal health services, particularly for rural households, but diagnostic testing is uncommon and first-line artemisinin combination therapy (ACT) can be expensive. In the absence of publicly-funded ACT subsidies, fewer than $15 \%$ of fevers treated for malaria receive appropriate, effective therapy in the retail sector. ${ }^{6}$

Starting in 2010, phase 1 of the Global Fund Affordable Medicines Facility-malaria 
(AMFm) piloted private-sector subsidies for ACTs in eight countries: Cambodia, Ghana, Kenya, Madagascar, Niger, Nigeria, Tanzania (mainland and Zanzibar) and Uganda. $^{7}$ The pilot led to a drop in the retail prices of ACTs in most pilot countries below that of cheaper, ineffective drugs and substantial cost savings were seen by the end consumer. The retail market share of ACTs in Kenya jumped from $12 \%$ to $61 \%$ in the first 18 months of the programme. ${ }^{8}$

Yet the sharp reduction in ACT cost may have encouraged overuse of the drug. Malaria is not the cause of illness in a large proportion (36-77\%) of febrile patients seeking care within the retail sector. ${ }^{9}{ }^{10}$ In Tanzania, one study determined that the majority $(80 \%)$ of clients obtaining ACTs from retail shops were not in fact parasitemic, while parasitemic clients purchased ACTs only $69 \%$ of the time. ${ }^{11}$ This points to a clear need to improve targeting of antimalarials purchased in the retail sector. In 2012, the Global Fund revised the AMFm strategy and ended the stand-alone ACT subsidy. Instead, countries could incorporate wholesale drug subsidies into their malaria control portfolios and provide subsidised RDTs to the private sector using Global Fund grants. The Kenyan Ministry of Health continued the private sector subsidy, but not at the same level provided by the AMFm programme. As a result, while ACTs are still sold over the counter and are widely available in the private retail sector, the retail price of ACTs increased approximately threefold. ${ }^{12} 13$

Appropriate targeting of subsidised ACTs could be improved by adding confirmatory testing to the programme. Using conditional subsidies for ACTs, dependent on a positive diagnostic test, could further enhance targeting and sustainability of the subsidy. The hypothesis is that such a strategy would reduce ACT overuse. In theory, this could be achieved using point-of-care malaria rapid diagnostic tests (RDTs). Evidence from several settings in Africa shows that, with appropriate training and supervision, laypersons can safely and correctly use RDTs for community diagnosis. ${ }^{10}{ }^{11} 11^{15}$ Yet the potential impact of incorporating diagnostic testing into an ACT subsidy programme operating within the retail sector remains largely unknown.

To address this evidence gap, we designed a publicprivate partnership (PPP) intervention to target antimalarials at those with confirmed malaria infection. This protocol paper describes the rationale and design of a cluster randomised controlled trial (c-RCT) in an area of Western Kenya with high malaria transmission. The goal of the c-RCT is to implement the PPP and to evaluate its impact on uptake of malaria testing and drug purchasing behaviour of febrile community members.

\section{Aims and objectives}

The overall objective of this study is to evaluate the public health impact of targeted antimalarial subsidies delivered through a partnership between CHVs and the private retail sector in Kenya. As part of the study, public-health sector CHVs offer and administer free RDTs to children over 1 year and adults with a fever in intervention communities. Conditional on the results of the test, those with a positive result are offered a voucher to purchase a subsidised WHO-qualified ACT at a participating local medicine retailer. The primary hypothesis is that this public-private partnership intervention will increase uptake of malaria testing before treatment and will lead to appropriate drug purchasing decisions after testing. The primary outcome is the proportion of people with fever that receive a malaria test (either RDT or microscopy) from any source (either CHVs or health facilities) at 12 months, which will be compared between the intervention and control arms.

\section{METHODS AND ANALYSIS}

The SPIRIT (Standard Protocol Items for Randomised Trials) recommendations were referenced in developing this protocol.

\section{Overall study design}

The study is being carried out in two subcounties in Western Kenya (Bungoma East and Kiminini) with similar malaria burden but different access to health services (figure 1). We used a stratified c-RCT design to assign all 32 eligible community units (CUs) in the area to either the intervention or control arm. A community unit is an existing administrative unit that averages about 1000 households ( $\sim 5000$ people). In order to be eligible for the study, a $\mathrm{CU}$ had to have an existing system of trained community health volunteers (CHVs) in place. In practice, each eligible $\mathrm{CU}$ has $\sim 20 \mathrm{CHVs}$ and one community health extension worker (CHEW) who supervises the work of the CHVs, although the number of households and active $\mathrm{CHV}$ ser $\mathrm{CU}$ varies from place to place. Since the structure of community health activities by CU entails CHVs serving only their home $\mathrm{CU}$, CUs were the natural choice for clusters in the c-RCT design: contamination between clusters is expected to be minimal. In the existing structure, CHVs are a volunteer workforce involved in short-term health campaigns and health promotion with no salary from the Ministry of Health. They occasionally receive remuneration for periodic donor-funded activities. Intervention-arm CHVs are reimbursed for study-related travel for supervision and the community-based organisations that they have established receive a small bonus twice per year.

In the intervention arm, CHVs offer household members free RDT testing and a voucher allowing the purchase of a qualified ACT at a reduced fixed price in the retail sector conditional on a positive test result. Individuals in the control arm receive usual care; they have access to standard CHV visits as per the Kenya Community Strategy Implementation guidelines. ${ }^{16}$ In practice, usual care means that control arm individuals decide whether they seek treatment in the formal sector 


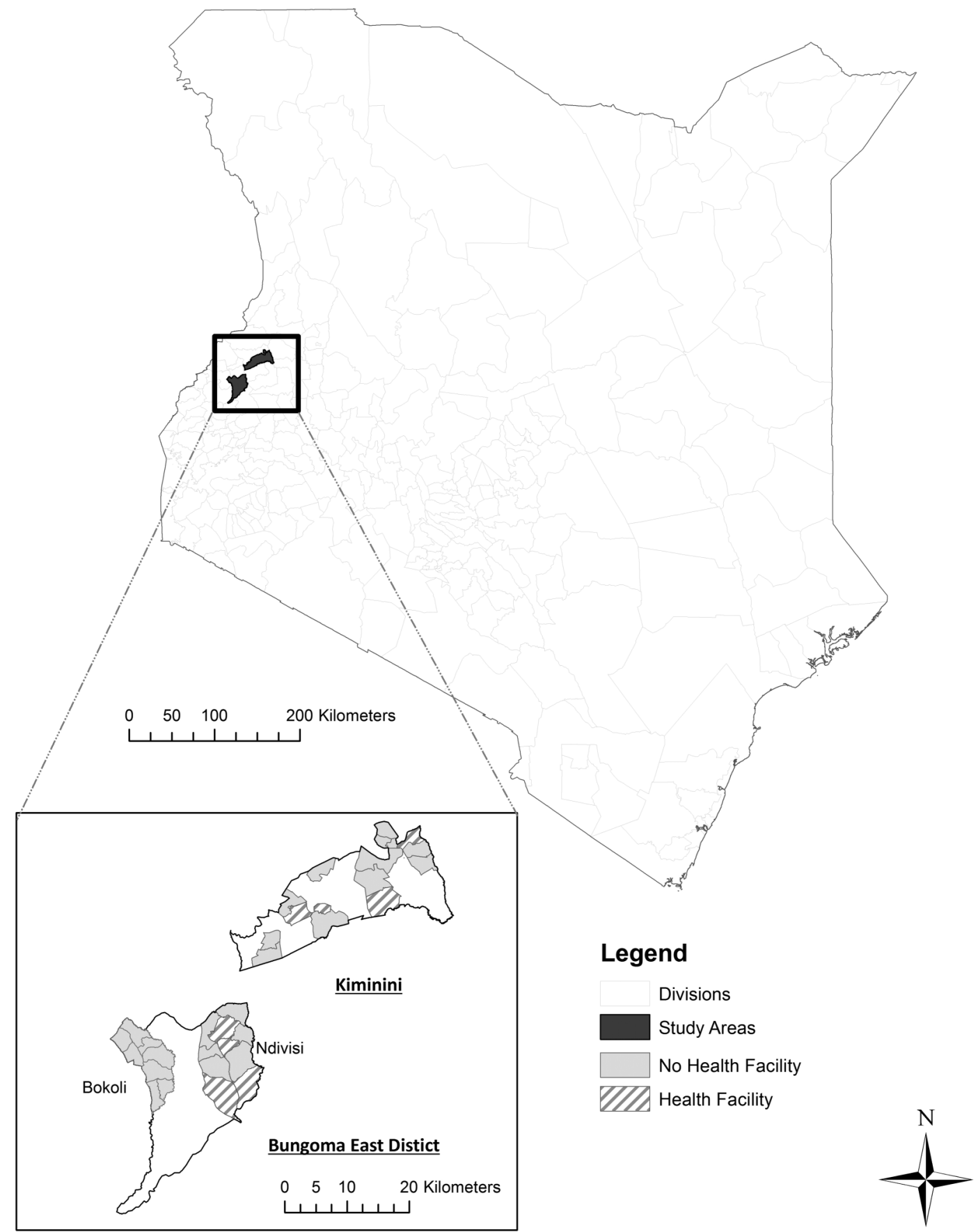

Figure 1 Study area and health facility access in selected community units.

(ie, testing and treatment via a health facility) or in the informal sector (ie, private retailers) in which no testing is currently available but where government-subsidised antimalarials are available.

Study outcomes and additional data are being collected via population-based cross-sectional household surveys of febrile individuals at four time points: baseline (from June 2015), 6, 12 and 18 months postbaseline. The households are selected randomly and independently of whether they received service from a CHV. Data obtained from these population-based surveys will be used to evaluate the impact of the community intervention. The intervention strategy is summarised in figure 2.

We chose a repeated cross-sectional survey c-RCT design rather than a cohort c-RCT design (in which a cohort of individuals is tracked over time) because we are interested in the population-level effects of the intervention on febrile individuals and the repeated crosssectional design is a natural choice for such research questions. ${ }^{17}$ If we had chosen a cohort design, we would have had to recruit both febrile and non-febrile individuals at baseline to be followed over time in order to identify sufficient febrile individuals at each follow-up 
Figure 2 Diagram of intervention strategy. CHWs, community health workers.

Intervention

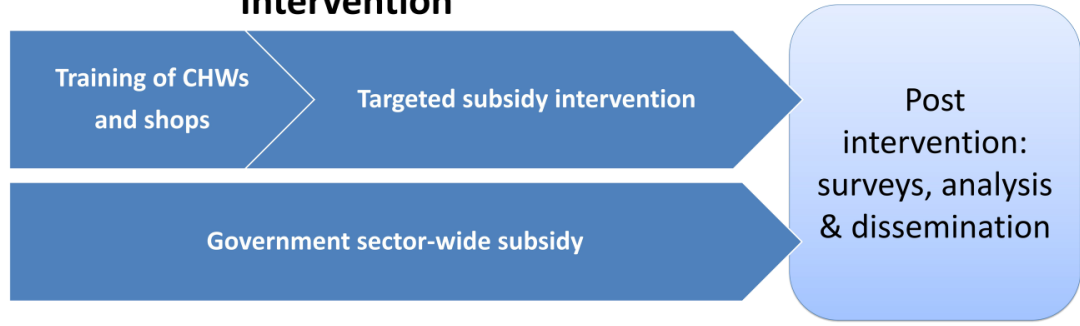

Comparison

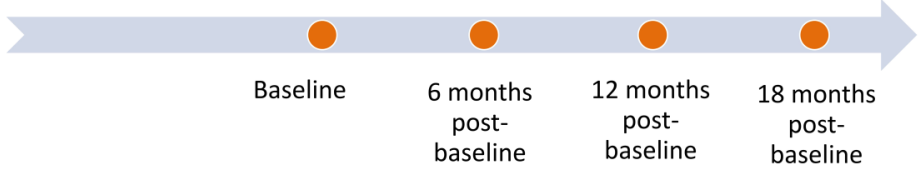

$=$ Household survey for eligible febrile individuals

time point. This would have been prohibitively expensive due to the large required sample size.

\section{Interventions}

The PPP intervention to target antimalarials at those with confirmed malaria infection was developed based on results from an earlier study we conducted in the study region. ${ }^{18}$ The study was a $2 \times 2$ factorial individually randomised trial whose goal was to test the use of a voucher system with a conditional ACT subsidy to improve uptake of testing and to determine the preferred subsidy levels of both the ACT and the RDT. $^{18}$ We evaluated two levels of RDT prices (free vs US $\$ 0.5$ cost to the client) and two levels of the conditional ACT subsidy (no subsidy vs US\$0.6 subsidy per dose). Findings from that trial indicate that (1) uptake of testing across all sources is improved when offered through CHVs compared to health facilities alone, (2) fewer clients chose to be tested when asked to pay for testing and (3) drug shops sometimes increased the base price of drugs for clients presenting a voucher, effectively reducing or negating the intended discount. On the basis of this evidence, we decided to offer free RDTs and, conditional on a positive result, a voucher for a fixed reduced price ACT rather than a fixed discount for an ACT. Intervention enrolment of the current c-RCT started in late July 2015. As in our earlier study, the RDTs are offered by study-trained CHVs and the ACTs are available for purchase at local retailers. The CareStart Malaria HRP-2 Pf RDT is used for all community-based testing, since it has been shown to have an average sensitivity and specificity of $94.8 \%$ and $95.2 \%$, respectively, for Plasmodium falciparum infection when compared to light microscopy. ${ }^{19}$

In the public component of the PPP intervention, all CHVs in the intervention CUs were invited for training on how to test for malaria using RDTs. The training was based on a validated 3-day Kenya Ministry of Health curriculum in conjunction with practical, skills oriented sessions. They were also taught how to take an axillary temperature using a digital thermometer and to recognise danger signs according to the WHO training module on malaria control case management guidelines. $^{20}$ Only CHVs who demonstrated competence during the theoretical and practical assessment were invited to participate in the intervention. The study team is monitoring $\mathrm{CHVs}$ through supervisory visits, initially every 2 weeks for the first 3 months of implementation, then monthly thereafter. CHVs requiring support are provided additional on-the-job training when necessary.

Clients who meet all of the following inclusion criteria are eligible to participate in the intervention (ie, given the opportunity to receive a CHV-administered RDT and conditional voucher): (1) age $\geq 1$ year, (2) self-reported fever or history of fever or feeling unwell with a malarialike illness within the past 2 days or axillary temperature above $37.5^{\circ}$ and, (3) consent from the client or their parent/legal guardian (if under 18 years). See English version of the consent forms in online supplementary material. The exclusion criteria include signs of severe disease or other problems requiring immediate referral to a health facility, or if the client has already visited a health facility or has already taken or purchased antimalarials for the current illness.

In the intervention arm, the CHV administers an RDT to each eligible participant who presents with a fever or malaria-like symptoms at any point during the 18-month implementation period. All participants receive a paper form with the results of their RDT clearly stated. Those with a negative RDT are advised to visit a health facility with documented test results. The negative RDT cassettes are collected by the study team during supervisory visits. Any individual with danger signs is referred to a facility regardless of RDT results. All of the government health facilities serving the intervention CUs have been sensitised about the study and asked to consider the results of the RDT in clinical management of the 
Table 1 Pricing scheme for voucher holders compared to standard retail prices

\begin{tabular}{lll}
\hline Age group & $\begin{array}{l}\text { Average } \\
\text { unsubsidised } \\
\text { price }\left(\text { KES }^{*}\right)\end{array}$ & $\begin{array}{l}\text { Study-subsidised } \\
\text { price for voucher } \\
\text { holders }\left(K^{*}\right)\end{array}$ \\
\hline $\begin{array}{l}\text { Adult dose } \\
\text { (>15 years) }\end{array}$ & $100-120$ & 40 \\
9-15 years & 80 & \\
3-8 years & 50 & 20 \\
1-2 years & 40 & 15 \\
$<1$ year & - & 10 \\
\hline${ }^{*}$ KES: Kenya Shilling; US\$1=KES100.8 (Central Bank of Kenya, \\
May 2016).
\end{tabular}

patient (ie, treating with ACT if positive, repeating test only when microscopy is available).

In the private component of the PPP intervention, a participant who has a positive RDT is given a serialised voucher for a WHO-approved quality-assured ACT to be purchased at a participating drug shop at a reduced fixed price according to the age-specific dosage required (table 1). The voucher is valid for 3 days from the date of issue. The holder may redeem the voucher by providing both the voucher and the positive RDT cassette to the participating medicine outlet to enable verification. Both are collected by the study team in return for payment of the difference between the normal retail price and the voucher price plus 5 Kenya shillings (KES). A woman with a positive RDT who the CHV determines may be pregnant (based on client self-report of last menstrual period) would not be offered a voucher, but would instead receive a referral to a health facility.

\section{Setting}

Kiminini subcounty covers an area of $395.3 \mathrm{~km}^{2}$ with an estimated population of 231191 , of which $40 \%$ is covered by the study. About $18 \%$ of the population does not have formal education, and $39.9 \%$ lives below the poverty line (County Government of Trans Nzoia, 2013). Bungoma East subcounty covers an area of $404.4 \mathrm{~km}^{2}$ with an estimated population of 260150 , of which $35 \%$ is covered by the study. About $14 \%$ of the population does not have a formal education, and 53\% lives below the poverty line (County Government of Bungoma, 2013). Both subcounties have a similar malaria burden, predominantly $P$. falciparum with perennial transmission. The study population consists of all individuals resident in the 18 eligible community units in Bungoma East subcounty and the 14 eligible community units in Kiminini subcounty.

\section{Randomisation}

The study area is naturally divided into three distinct geographic areas: Bokoli, Ndivisi (both in Bungoma East subcounty) and Kiminini with 8, 10 and 14 CUs, respectively (figure 1). Since four of the CUs in each of Ndivisi and Kiminini have health facilities with laboratories that perform malaria testing, the three areas are then naturally divided into five strata, each of which has an even number of CUs. Stratified randomisation of the 32 CUs within these 5 strata was used in order to reduce the probability of baseline imbalances in the outcomes due to differential access to healthcare. Randomisation was performed by the lead statistician (ELT) using Stata SE V.14.O Software (College Station: Texas: Statcorp LP). Half of the community units within each strata were randomly allocated to the intervention arm so that half of the CUs in each subcounty were in the intervention arm (9 in Bungoma East subcounty and 7 in Kiminini subcounty).

\section{Sensitisation and recruitment}

The CHVs, CHEWs, local health management teams, village elders and chiefs facilitated sensitisation about the intervention. Most households are already familiar with their local CHV and anyone feeling ill with a malaria-like illness is advised to contact the CHV.

All retail medicine shops serving the intervention clusters were identified through a comprehensive census and invited to participate. A total of 36 shops have been enrolled across both subcounties. The shop owners and attendants have been trained on current Government of Kenya malaria treatment guidelines, ${ }^{21}$ the role of RDTs in case management and the study procedures. They are encouraged to stock quality-assured ACTs at the normal government-subsidised price. Prior to participation, the shop owners sign an informed consent and a terms of reference document.

\section{Outcomes}

All study outcomes will be measured in individuals who have experienced a febrile illness in the previous 4 weeks. These outcomes will be ascertained through four cross-sectional household surveys (baseline, 6, 12 and 18 months). While outcome assessors cannot be blinded to the study arm due to the unblinded nature of the trial, data analysts will be blinded to the study arm. The primary outcome is the proportion of fevers that receives a malaria test (either RDT or microscopy) from any source (either CHVs or health facilities). The main secondary outcome is the proportion of all ACTs taken by people with a malaria positive test. Additional secondary outcomes are: the proportion of all ACTs taken by people without a test, the proportion of those with a positive test who get an ACT and the proportion of those with a negative test who get an ACT. We will also compare drug adherence among those who redeem a voucher for their ACT and those who pay the retail price. 'Appropriate ACT use' is defined as "taking ACT if positive or not taking ACT if negative among those who took a malaria test" and 'targeted ACT use' is defined as "taking ACT if positive or not taking ACT if negative among all participants". ${ }^{18}$ 
Additional data are collected for intervention participants by the CHV who record temperature, participant age and RDT results, as well as client follow-up information (health condition and actions taken) for each participant 4 days after testing.

Figure 3 shows the expected behaviour of febrile study participants in the intervention and control arms. We assume that this is the same at each of the three follow-up time points.

\section{Survey procedures}

Study outcomes and additional data will be collected via the four household surveys. Each survey round will take $\sim 2$ months to complete across all 32 CUs. To meet eligibility criteria, respondents must (1) reside in a study CU, (2) be older than 1 year and (3) report a history of a malaria-like illness among at least one household member within the past 4 weeks. Information about children $<18$ years is obtained by interviewing the parent/ guardian.

For only one reported fever per household for the previous 4-week period, the survey team records the type and source of any $\operatorname{drug}(\mathrm{s})$ taken, and self-reported test results of any diagnostic test for malaria (RDT or microscopy) performed prior to treatment. If there is more than one individual with a history of fever, an adult is selected over a child, or if both are adults or children, selection is based on the alphabetical order of the given names. When made available, relevant drug packaging and test records are reviewed to reduce recall bias. Survey teams also have examples of antimalarial drug packaging to help with identification.

\section{Sample size calculation}

There are two distinct target sample sizes in this study. First, the number of eligible respondents (ie, who report on action taken for fever either by themselves or by a household member); second, the number of individuals (households) surveyed in order to meet the target number of eligible respondents.

The target sample size of eligible respondents is 640 / arm at each time point (40 in each of the $32 \mathrm{CU}, 1280$ in total). Power is based on a cluster randomised twosample, two-tailed t-test for the comparison of two proportions at a single time point using standard formulae, which use the coefficient of variation (CV) as the measure of between-cluster variability. ${ }^{23}$ To ensure that our overall two-tailed type I error $(\alpha)$ was $5 \%$, we fixed the $\alpha$ level at $1.667 \%$ (ie, $5 \% / 3$ ) for each of the three follow-up time points $(6,12$ and 18 months postbaseline), using the conservative Bonferroni correction..$^{22}$ To further protect against other possible losses in power, we conservatively based the power on a matched-cluster design which includes a larger design penalty than the stratified-design sample size calculator for the same assumed level of clustering. ${ }^{23}$ Assumptions on effect sizes, CV and ICC for the primary and secondary outcomes are shown in table 2. Each $\mathrm{CV}$ was estimated using a published strategy: ${ }^{23} \mathrm{CU}$-specific proportions were assumed to be normally distributed, centred on the control arm proportion with SD derived from an assumed range for $95 \%$ of the CU-specific proportions (ie, for a width of $\sim 4 \mathrm{SD}$ ). Conservative estimates for the range were used as follows: $50 \%$ for the primary outcome and for the proportion of ACTs taken by those with no test; $25 \%$ for the proportion of ACTs taken by those with a positive test; and $10 \%$ for the proportion that takes ACT after a positive test and for the proportion that takes ACT after a negative test. Using the assumed CVs, the ICC values were then estimated using the following formula: ICC $=\mathrm{CV}^{2} \times \pi /(1-\pi)$, where $\pi$ is the assumed control-arm proportion for the outcome. ${ }^{23}$

On the basis of pilot data from Bungoma, we hypothesised an increase from $31 \%$ in control to $70 \%$ in intervention in our primary outcome of uptake of testing (table 2 and figure 3). With a target sample size of 640 eligible respondents per arm (40 in each of the $32 \mathrm{CU}$, 1280 in total) at each time point and assuming a conservative ICC estimate of 0.073 (corresponding to a $\mathrm{CV}$ of 0.40 ), we will have more than $95 \%$ power to detect this hypothesised effect size. On the basis of our earlier work, we assume that the PPP intervention increases both uptake of testing (from $31 \%$ to $70 \%$ ) and the proportion that takes ACT after a positive test (from $70 \%$ to

\section{Intervention}

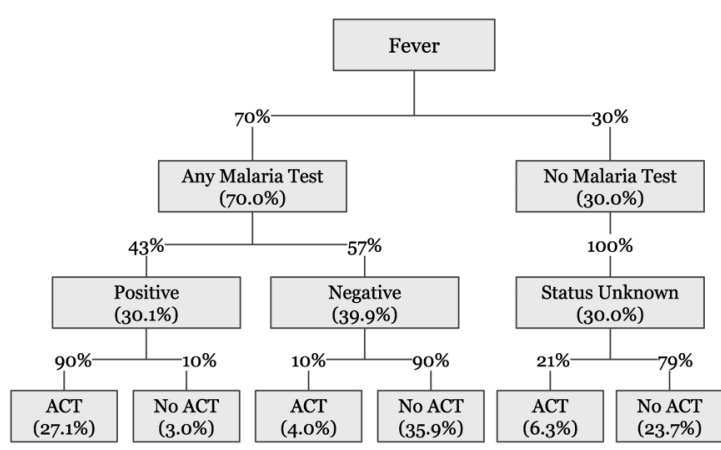

Control

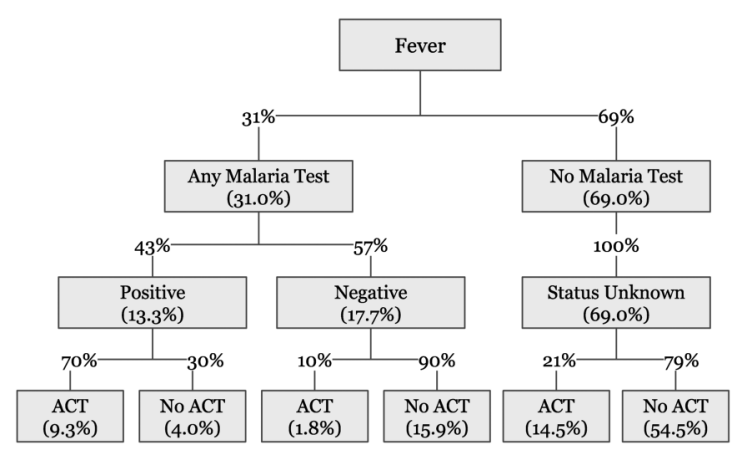

Figure 3 Expected behaviour and test results of febrile study participants in the intervention and control arms. ACT, artemisinin combination therapy. 
Table 2 Summary of assumed intervention effects, clustering and power for primary and secondary outcomes

\begin{tabular}{|c|c|c|c|c|c|}
\hline Outcome & $\begin{array}{l}\text { Intervention } \\
\text { vs Control }\end{array}$ & $\mathrm{CV}^{*}$ & ICC* & $\begin{array}{l}\text { Assumed n per } \\
\text { cluster }^{\dagger}\end{array}$ & Power ${ }^{\ddagger}$ \\
\hline \multicolumn{6}{|l|}{ Primary } \\
\hline Proportion of fevers with test ${ }^{\S}$ & $70 \%$ vs $31 \%$ & 0.40 & 0.073 & 40 & $98 \%$ \\
\hline \multicolumn{6}{|l|}{ Secondary } \\
\hline Proportion of ACT taken by those who test positive ${ }^{\S}$ & $72.5 \%$ vs $36.5 \%$ & $0.17^{\star *}$ & $0.017^{\star \star}$ & 10 & $>99 \%$ \\
\hline Proportion of ACT taken by those with no test ${ }^{\S}$ & $16.9 \%$ vs $56.6 \%$ & 0.22 & 0.064 & 10 & $>99 \%$ \\
\hline Proportion who take ACT after a positive test ${ }^{\S}$ & $90 \%$ vs $70 \%$ & $0.04^{\star \star}$ & $0.003^{\star \star}$ & 5 & $N A^{\dagger \dagger}$ \\
\hline Proportion who take ACT after a negative test ${ }^{\S}$ & $10 \%$ vs $10 \%$ & 0.25 & 0.007 & 7 & $\mathrm{NA}^{\dagger \dagger}$ \\
\hline \multicolumn{6}{|c|}{ 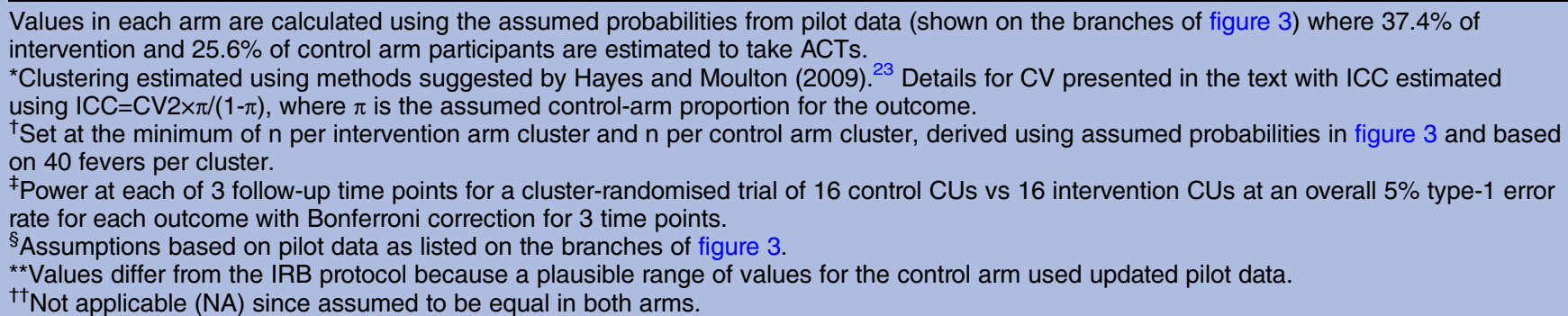 } \\
\hline $\begin{array}{l}\text { ACT, artemisinin combination therapy; CU, community units; } \\
\text { Institutional Review Board. }\end{array}$ & , coefficient of variati & $\mathrm{n}$; ICC, in & aclass corre & ation coefficient; IRB, & \\
\hline
\end{tabular}

$90 \%)$, but that there is no effect on the proportion of positive test results $(43 \%)$ or on ACT purchasing behaviour conditional on a negative test (10\% in both arms) or for those with no test $(21 \%$ in both arms, table 2 and figure 3). On the basis of these assumptions, the assumed effect sizes for the secondary outcomes are derived together with the sample sizes. In particular, it is estimated that the intervention will increase the proportion of ACT taken that is taken by those with a positive test from $36.5 \%$ to $72.5 \%$ and will decrease the proportion of ACT taken by those with no test from $56.6 \%$ to $16.9 \%$ with excellent power for both outcomes (table 2). On the basis of our a priori assumptions, the intervention is not expected to have an effect on the two secondary outcomes of ACT purchasing behaviour after a negative test or no test. Overall, $37.4 \%$ of intervention participants and $25.6 \%$ of control participants are expected to take ACT (figure 3). For example, in the intervention arm, this is based on the $27.1 \%$ of all participants who are positive and take ACT, the $4 \%$ who are negative and take ACT, and the $6.3 \%$ without a test who take ACT.

For the second target sample size, in order to obtain the required sample size of 1280 febrile individuals, we assume that we will need to sample a total of 5766 individuals (2883 per arm) at each of the 3 follow-up surveys. This is based on an assumed population 4-week fever prevalence of $22.2 \%$, which we derived assuming: under 5 years 4-week fever prevalence of $35.5 \%$ (our preliminary work showed: $33 \%$ in Bungoma East and $38 \%$ in Kiminini); fever incidence in older children and adults is roughly half of that in under 5 years; and a population mix of 1:3 for under 5 years versus older children and adults.

\section{Data management}

Data about intervention participants and their RDT results are being collected by CHVs using customised carbonless-copy client registers designed to be read and digitised by Captricity for automated data entry. Data are routinely scanned and digitised by field supervisors.

The household survey data are collected on encrypted, password protected Android netbooks running the OpenDataKit platform. Internal consistency checks and data quality checks are programmed into the forms as well as evaluated by data managers. When in the field, netbooks are locked in a secure cabinet nightly and data are removed from the tablets several times per week. Only deidentified data are shared with data analysts. On completion of the trial and publication of trial findings, the final trial data set may be requested from the authors.

\section{Data analysis}

All study outcomes are individual-level binary outcome measures. Estimated intervention effects will be reported with $95 \%$ CIs. We will compare each of the study outcomes between study arms at each time point. Analyses will be based on the intention-to-treat principle. We will use a mixed effects modelling approach with a random intercept for each CU (to account for clustering) and fixed effects for strata (to account for the stratified design). Models with adjusted and unadjusted estimates will be presented for all analyses. Adjustment covariates will include: age, sex, household size and household socioeconomic status. Sensitivity analyses will be conducted if other relevant covariates are identified. Analyses may be adjusted for the baseline level of the outcome variable in order to improve the precision of 
estimation of the intervention effect. Owing to the repeated cross-sectional c-RCT design, we will not need to account for missing data due to attrition of study participants. Instead, survey non-response may lead to nonrepresentativeness of the cross-sectional samples. We will describe the non-response rates and compare them between arms. Given our prior experience in the region, non-response is anticipated to be minimal and to be comparable between arms.

Autonomy of testing and treatment decisions depends on the age of the febrile individual and potentially other subgroups. We will evaluate each subgroup effect by testing for an interaction effect between the covariate (eg, age) and treatment arm. If there is evidence of different intervention effects by subgroup, we will report results separately for each subgroup. Subanalyses will be performed to determine the sensitivity of results to the precise definition of the outcome. The first set of subanalyses will look at the set of main outcomes using only malaria tests for which documentation was provided to the data collectors.

Our primary aim is to determine whether there is a significant difference between the two study arms in the proportion of clients with fever who are tested prior to any treatment after adjusting for relevant covariates at each of the follow-up periods. We will use the Benjamini Hochberg procedure for determining significance of the three tests of the difference between groups at each follow-up time point. ${ }^{24}$ We will also compare secondary measures using the same modelling and adjustment approach.

\section{Process evaluation and monitoring}

The study team visits participating retail outlets several times each month to monitor availability of ACTs and prices of antimalarials. The team cross-checks redeemed vouchers with the used positive RDTs. To facilitate implementation monitoring, CHVs and retail outlets in the intervention arm routinely provide records, the study team rereads used RDTs, and supervisors conduct unannounced retail outlet visits and observations of CHV testing.

A process evaluation plan has also been developed to provide a holistic strategy for monitoring and describing the context of intervention implementation, as well as to provide insight into opportunities for improvement, replication and potential for a further scale up. We have explicitly identified the mechanisms for systematically tracking and summarising the key inputs, activities and outputs of the intervention in order to assess inherent assumptions, penetration, unintended spillover and fidelity of project implementation to the protocol. We have also identified and will develop tools to address information gaps, in particular with regard to gathering information on participating CHVs' and drug shop attendants' perspectives on feasibility, acceptability and motivation related to their intervention roles.

\section{Informed consent}

CHVs obtain written consent from eligible clients before testing. For minors (under 18 years of age), a parent or legal guardian is asked to provide consent, along with verbal assent from minors over the age of 8 years.

Household survey respondents provide verbal consent. Shop owners of participating retail medicine outlets are asked to provide written informed consent. For shops that choose not to participate in the voucher scheme, we seek verbal consent to collect only study-related survey data such as stocking and sales of antimalarials.

Both the Duke University Medical Center Institutional Review Board and Institutional Research and Ethics Committee ethical approval bodies granted waivers for documentation of informed consent for both the household survey and retail outlet survey participants; verbal consent was sought from potential participants in these activities. Waivers were granted on the grounds that for both activities, the consent document would be the only information linking the respondent to his or her study ID and the surveying presents no more than minimal risk to the client and does not include any procedure for which consent would normally be required outside of the research setting.

\section{Trial oversight}

The trial management committee (TMC) includes all coauthors of this protocol paper, with leadership provided by the study principal investigator, Dr O'Meara. Other specific roles are CHV oversight (Dr Menya), retail sector liaison and oversight (Dr Laktabai), data analysis oversight (Dr Turner), data management and data analysis (Ms Platt), study coordination (Ms Lesser) and advisory roles on economics (Dr Mohanan and Ms Maffioli). The TMC meets weekly to monitor field activities. No data safety and monitoring committee was created since the rapid diagnostic tests administered by CHVs are routinely used in a range of different settings and are considered safe when used by those carefully trained to use them, such as the CHVs in our study. Since no interim analyses are planned and data quality is monitored by the TMC through (blinded) periodic reports, no data monitoring committee was created.

\section{Dissemination}

Findings from this study will be shared through stakeholder meetings including with the Kenyan Ministry of Health, peer-reviewed publications and conference presentations. Results will also be reported through clinicaltrials.gov.

\section{Author affiliations}

${ }^{1}$ Moi School of Medicine, Eldoret, Kenya

${ }^{2}$ Duke Global Health Institute, Duke University, Durham, North Carolina, USA

${ }^{3}$ Department of Biostatistics and Bioinformatics, Duke University, Durham,

North Carolina, USA

${ }^{4}$ Department of Economics, Duke University, Durham, North Carolina, USA

${ }^{5}$ Sanford School of Public Policy, Duke University, Durham, North Carolina, USA 
${ }^{6}$ Moi University School of Public Health, College of Health Sciences, Eldoret, Kenya

${ }^{7}$ Division of Infectious Diseases and International Health, Duke University Medical Center, Durham, North Carolina, USA

Acknowledgements The authors thank Dr Indrani Saran for her valuable comments on a draft of this manuscript and Ms Paige Meier for her editorial assistance with references, figures and formatting.

Contributors JL, AL, AP and ELT drafted the manuscript. All authors contributed to the study design. All authors read and approved of the final version of the manuscript.

Funding This work is supported by the National Institutes of Health-National Institute of Allergy and Infectious Diseases (NIH-NIAID) grant number R01Al110478. The content is solely the responsibility of the authors and does not necessarily represent the official views of the National Institute of Allergy and Infectious Diseases or the National Institutes of Health. The study sponsor had no influence on the study design; data collection, analysis or interpretation; content of the manuscript, nor the authors' decision to submit this manuscript. The researchers operated independently from the funder in these matters. All authors had full access to all data and take responsibility for the integrity and accurate analysis of the data.

Competing interests All authors have completed the ICMJE uniform disclosure form at http://www.icmje.org/coi_disclosure.pdf and declare: all authors had financial support from the National Institute of Allergy and Infectious Diseases of the National Institutes of Health (US) for the submitted work.

Ethics approval Moi University School of Medicine Institutional Research and Ethics Committee (approval number 0001403) and the Duke University Medical Center Institutional Review Board (Pro00063384). The study has also been registered on clinicaltrials.gov (NCT02461628).

Provenance and peer review Not commissioned; externally peer reviewed.

Open Access This is an Open Access article distributed in accordance with the Creative Commons Attribution Non Commercial (CC BY-NC 4.0) license, which permits others to distribute, remix, adapt, build upon this work noncommercially, and license their derivative works on different terms, provided the original work is properly cited and the use is non-commercial. See: http:// creativecommons.org/licenses/by-nc/4.0/

\section{REFERENCES}

1. Abuya TO, Mutemi W, Karisa B, et al. Use of over-the-counter malaria medicines in children and adults in three districts in Kenya: implications for private medicine retailer interventions. Malar $J$ 2007;6:57.

2. Amin AA, Marsh V, Noor AM, et al. The use of formal and informal curative services in the management of paediatric fevers in four districts in Kenya. Trop Med Int Health 2003;8:1143-52.

3. Chuma J, Abuya T, Memusi D, et al. Reviewing the literature on access to prompt and effective malaria treatment in Kenya: implications for meeting the Abuja targets. Malar J 2009; 8:243.

4. Malik EM, Hanafi $\mathrm{K}$, Ali SH, et al Treatment-seeking behaviour for malaria in children under five years of age: implication for home management in rural areas with high seasonal transmission in Sudan. Malar J 2006:5:60.
5. Littrell M, Gatakaa H, Evance I, et al. Monitoring fever treatment behaviour and equitable access to effective medicines in the context of initiatives to improve ACT access: baseline results and implications for programming in six African countries. Malar J 2011:10:327.

6. World Health Organization. World Malaria Report. Geneva, 2012(a).

7. World Health Organization. Q\&A on the Affordable Medicines Facility malaria (AMFm) Secondary Q\&A on the Affordable Medicines Facility malaria (AMFm). 2012(b). http://www.who.int/malaria/media/ affordable_medicines_facility_qa/en/

8. Tougher $\mathrm{S}, \mathrm{Ye} \mathrm{Y}$, Amuasi $\mathrm{JH}$, et al. Effect of the Affordable Medicines Facility-malaria (AMFm) on the availability, price, and market share of quality-assured artemisinin-based combination therapies in seven countries: a before-and-after analysis of outlet survey data. Lancet 2012;380:1916-26.

9. Mangham LJ, Cundill B, Achonduh OA, et al. Malaria prevalence and treatment of febrile patients at health facilities and medicine retailers in Cameroon. Trop Med Int Health 2012;17:330-42.

10. Cohen J, Dupas P, Schaner SG. Price subsidies, diagnostic tests, and targeting of malaria treatment: evidence from a randomized controlled trial. Am Econ Rev 2015;105:609-45.

11. Briggs MA, Kalolella A, Bruxvoort $\mathrm{K}$, et al. Prevalence of malaria parasitemia and purchase of artemisinin-based combination therapies (ACTs) among drug shop clients in two regions in Tanzania with ACT subsidies. PLoS One 2014;9:e94074.

12. ACTwatch PSla. Kenya Outlet Survey Findings 2010-2014. ACTwatch Research Brief. Washington DC, USA: ACTwatch Research Brief, 2015

13. Kioko U, Riley C, Dellicour S, et al. A cross-sectional study of the availability and price of anti-malarial medicines and malaria rapid diagnostic tests in private sector retail drug outlets in rural Western Kenya, 2013. Malar J 2016;15:359.

14. Hamer DH, Brooks ET, Semrau K, et al. Quality and safety of integrated community case management of malaria using rapid diagnostic tests and pneumonia by community health workers. Pathog Glob Health 2012;106:32-9.

15. Chanda $\mathrm{P}$, Hamainza $\mathrm{B}$, Moonga $\mathrm{HB}$, et al. Community case management of malaria using ACT and RDT in two districts in Zambia: achieving high adherence to test results using community health workers. Malar J 2011;10:158.

16. Republic of Kenya Ministry of Health. Community Strategy Implementation Guidelines for Managers of the Kenya Essential Package for Health at the Community Level. 2007. https://www.slideshare.net/chskenya/community-healthstrategyimplementationguide2007

17. Murray DM. Design and analysis of group-randomized trials. Oxford UK: Oxford University Press, 1998.

18. Prudhomme O'Meara W, Mohanan M, Laktabai J, et al. Assessing the independent and combined effects of subsidies for antimalarials and rapid diagnostic testing on fever management decisions in the retail sector: results from a factorial randomised trial in western Kenya. BMJ Global Health 2016;1:e000101.

19. Abba K, Deeks JJ, Olliaro P, et al. Rapid diagnostic tests for diagnosing uncomplicated $\mathrm{P}$. falciparum malaria in endemic countries. Cochrane Database Syst Rev 2011;(7):Cd008122.

20. World Health Organization. Training module on malaria control: case management. Malta: World Health Organization, 2012(c).

21. Republic of Kenya Ministry of Health. National guidelines for the diagnosis, treatment and prevention of malaria in Kenya. 4th edn, 2014.

22. Aickin M, Gensler $\mathrm{H}$. Adjusting for multiple testing when reporting research results: the Bonferroni vs Holm methods. Am J Public Health 1996;86:726-8.

23. Hayes RJ, Moulton LH. Cluster randomised trials. Boca Raton, FL: CRC Press, 2009.

24. Benjamini Y, Yekutieli D. The control of the false discovery rate in multiple testing under dependency. The Ann Stat 2001;29:1165-88. 